This item was submitted to Loughborough's Research Repository by the author.

Items in Figshare are protected by copyright, with all rights reserved, unless otherwise indicated.

\title{
Superlight small bipolarons in the presence of strong Coulomb repulsion
}

PLEASE CITE THE PUBLISHED VERSION

\section{LICENCE}

CC BY-NC-ND 4.0

\section{REPOSITORY RECORD}

Hague, J.P., P.E. Kornilovitch, J.H. Samson, and A.S. Alexandrov. 2019. "Superlight Small Bipolarons in the Presence of Strong Coulomb Repulsion”. figshare. https://hdl.handle.net/2134/2748. 


\title{
Superlight small bipolarons in the presence of strong Coulomb repulsion
}

\author{
J.P. Hague, ${ }^{1}$ P.E. Kornilovitch,${ }^{2}$ J.H. Samson,${ }^{1}$ and A.S. Alexandrov ${ }^{1}$ \\ ${ }^{1}$ Department of Physics, Loughborough University, Loughborough, LE11 3TU, United Kingdom \\ ${ }^{2}$ Hewlett-Packard Company, 1000 NE Circle Blvd, Corvallis, Oregon 97330, USA
}

(Dated: June 1, 2006)

\begin{abstract}
We study a lattice bipolaron on a staggered triangular ladder and triangular and hexagonal lattices with both long-range electron-phonon interaction and strong Coulomb repulsion using a novel continuous-time quantum Monte-Carlo (CTQMC) algorithm extended to the Coulomb-Fröhlich model with two particles. The algorithm is preceded by an exact integration over phonon degrees of freedom, and as such is extremely efficient. The bipolaron effective mass and bipolaron radius are computed. Lattice bipolarons on such lattices have a novel crablike motion, and are small but very light in a wide range of parameters, which leads to a high Bose-Einstein condensation temperature. We discuss the relevance of our results with current experiments on cuprate high-temperature superconductors and propose a route to room temperature superconductivity.
\end{abstract}

PACS numbers: 71.38.-k

As recognized by Landau, Pekar and Fröhlich, an electron may drag a lattice distortion as it moves through an ionic material, leading to a new particle - the polaron, which has quite different properties to the original electron (for reviews see, for example, Refs. [1, 2]). At weak coupling, two polarons can be bound into a large bipolaron via exchange forces, without assuming anything more complicated than the Fröhlich electron-phonon interaction [3]. On increasing density large bipolarons overlap, giving rise to either a conventional (BCS) superconductor or a normal metal. Electron-phonon interactions may overcome the Coulomb repulsion between electrons, so the resulting interaction becomes attractive at a distance of the order of the lattice constant 4]. Then two small polarons form tightly bound pairs, i.e. small bipolarons, in the strong electron-phonon coupling limit. Earlier studies [5] considered small bipolarons as entirely localized objects. However, a perturbation expansion with respect to the hopping integral has proved they are itinerant quasiparticles existing in Bloch states for any finite coupling with phonons and forming a Bose-Einstein condensate (BEC) of charge $2 e$ bosons at sufficiently low temperatures [6].

For very strong electron-phonon coupling, polarons become self-trapped on a single lattice site. The energy of the resulting small polaron is given as $E_{p}=-\lambda z t$, where $\lambda$ is the electron-phonon coupling constant, $t$ is the hopping parameter and $z$ is the coordination number. Expanding about the atomic limit in small $t$ (which is small compared to $E_{p}$ in the small polaron regime, $\lambda>1$ ) the polaron mass is computed as $m^{*}=m_{0} \exp (\gamma z \lambda / \hbar \omega)$, where $\omega$ is the frequency of Einstein phonons, $m_{0}$ is the rigid lattice band mass, and $\gamma$ is a numerical constant. For the Holstein model [7], which is purely site local, $\gamma=1$. Bipolarons are on-site singlets in the Holstein model and their mass $m_{H}^{* *}$ appears only in the second order of $t[\underline{6}]$ scaling as $m_{H}^{* *} \propto\left(m^{*}\right)^{2}$ in the limit $\hbar \omega \gg \Delta$ , and as $m_{H}^{* *} \propto\left(m^{*}\right)^{4}$ in a more realistic regime $\hbar \omega \ll \Delta$
[4]. Here $\Delta=2 E_{p}-U$ is the bipolaron binding energy, and $U$ is the on-site (Hubbard) repulsion. Since the Hubbard $U$ is about $1 \mathrm{eV}$ or larger in strongly correlated materials, the electron-phonon coupling must be large to stabilize on-site bipolarons and the Holstein bipolaron mass appears very large, $m_{H}^{* *} / m_{0}>1000$, for realistic values of phonon frequency.

This estimate led some authors to the conclusion that the formation of itinerant small polarons and bipolarons in real materials is unlikely [8], and high-temperature bipolaronic superconductivity is impossible [9]. However, one should note that the Holstein model is an extreme polaron model, and typically yields the highest possible value of the (bi)polaron mass in the strong coupling limit. Many advanced materials with low density of free carriers and poor mobility (at least in one direction) are characterized by poor screening of high-frequency optical phonons and are more appropriately described by a long-range Fröhlich electron-phonon interaction 4]. For a long-range Fröhlich interaction the parameter $\gamma$ is less than 1 ( $\gamma \approx 0.3$ on the square lattice and $\gamma \approx 0.2$ on the triangular lattice 10,11|), reflecting the fact that in a hopping event the lattice deformation is partially preexistent. Hence the unscreened Fröhlich electron-phonon interaction provides relatively light small polarons, which are several orders of magnitude lighter than small Holstein polarons. This has been confirmed by numerical Monte-Carlo simulations 11, 12], Lanczos diagonalization [13] and variational calculations [14].

This unscreened Fröhlich interaction combined with on-site repulsive correlations can also bind holes into intersite mobile bipolarons [10, 15]. Using an advanced variational method Bonča and Trugman [14] studied the chain model of Ref. [12] with two electrons for nearestneighbor e-ph interaction and a Hubbard $U$. Intersite bipolarons of Ref. 14] propagate along the chain with a mass which is still of the second order in the polaron mass as in the Holstein model. 
Here we study a bipolaron on a staggered triangular ladder (1D), triangular (2D) and strongly anisotropic hexagonal (3D) lattices using a continuous-time quantum Monte-Carlo technique. On such lattices, bipolarons are found to move with a crab like motion (Fig. 1(b)), which is distinct from the crawler motion (Fig. 1(c)) found on cubic lattices 6, 14]. Such bipolarons are small but very light for a wide range of electron-phonon couplings and phonon frequencies.

We use a generic Coulomb-Fröhlich model of electronphonon interactions which has the following Hamiltonian,

$$
\begin{aligned}
H & =-t \sum_{\left\langle\mathbf{n n}^{\prime}\right\rangle \sigma} c_{\mathbf{n}^{\prime} \sigma}^{\dagger} c_{\mathbf{n} \sigma}+\sum_{\mathbf{n n}^{\prime} \sigma} V\left(\mathbf{n}, \mathbf{n}^{\prime}\right) c_{\mathbf{n} \sigma}^{\dagger} c_{\mathbf{n} \sigma} c_{\mathbf{n}^{\prime} \sigma}^{\dagger} c_{\mathbf{n}^{\prime} \bar{\sigma}} \\
& +\sum_{\mathbf{m}} \frac{\hat{P}_{\mathbf{m}}^{2}}{2 M}+\sum_{\mathbf{m}} \frac{\xi_{\mathbf{m}}^{2} M \omega^{2}}{2}-\sum_{\mathbf{n} \mathbf{m} \sigma} f_{\mathbf{m}}(\mathbf{n}) c_{\mathbf{n} \sigma}^{\dagger} c_{\mathbf{n} \sigma} \xi_{\mathbf{m}} .
\end{aligned}
$$

Each vibrating ion has one phonon degree of freedom $\xi_{\mathbf{m}}$ associated with a single atom. The sites are numbered by the indices $\mathbf{n}$ or $\mathbf{m}$ for electrons and ions respectively. Operators $c$ annihilate electrons. The phonon subsystem is a set of independent oscillators with frequency $\omega$ and mass $M$. Here $\left\langle\mathbf{n n}^{\prime}\right\rangle$ denote pairs of nearest neighbors, and $\hat{P}_{\mathbf{m}}=-i \hbar \partial / \partial \xi_{\mathbf{m}}$ is the ion momentum operator. Coulomb repulsion $V\left(\mathbf{n}-\mathbf{n}^{\prime}\right)$ is screened up to the first nearest neighbors, with on site repulsion $U$ and nearest-neighbor repulsion $V_{C}$. In contrast, the Fröhlich interaction is assumed to be long-range, due to unscreened interaction with c-axis high-frequency phonons [4]. The form of the interaction with c-axis polarized phonons is specified via the force function [12], $f_{\mathbf{m}}(\mathbf{n})=\kappa\left[(\mathbf{m}-\mathbf{n})^{2}+1\right]^{-3 / 2}$, where $\kappa$ is a constant.
The dimensionless electron-phonon coupling constant $\lambda$ is defined as $\lambda=\sum_{\mathbf{m}} f_{\mathbf{m}}^{2}(0) / 2 M \omega^{2} z t$ which is the ratio of the polaron energy at $t=0$ to the kinetic energy of the free electron $z t$.

In the limit of high phonon frequency $\hbar \omega \gg t$ and large on-site Coulomb repulsion, the model is reduced to an extended Hubbard model with intersite attraction and suppressed double-occupancy [15]. Then the Hamiltonian can be projected onto the subspace of nearest neighbor intersite crab bipolarons. In contrast with the crawler bipolaron, the crab bipolaron's mass scales linearly with the polaron mass $\left(m^{* *}=4 m^{*}\right.$ on the staggered chain and $m^{* *}=6 m^{*}$ on the triangular lattice). Here we formulate the following question: Can such a bipolaron exist for more realistic intermediate values of the electron-phonon coupling and phonon frequency?

To answer this question, we have extended the CTQMC algorithm 11, 12, 16, 17] to systems of two particles with strong electron-phonon interactions. We have solved the bipolaron problem on a staggered ladder (Fig.1), triangular and anisotropic hexagonal lattices from weak to strong coupling in a realistic parameter range where usual limiting approximations fail.

The CTQMC method employed here has been described in detail with regard to the single polaron problem in Refs. 11, 16, 17]. Here we give a quick overview of the extended algorithm. The initial step is to determine the effective bipolaron action that results when the phonon degrees of freedom have been integrated out analytically. The action is a functional of two polaron paths in imaginary time which form the bipolaron and is given by the following double integral,

$$
\begin{aligned}
A[\mathbf{r}(\tau)] & =\frac{z \lambda \bar{\omega}}{2 \Phi_{0}(0,0)} \int_{0}^{\bar{\beta}} \int_{0}^{\bar{\beta}} d \tau d \tau^{\prime} e^{-\bar{\omega} \bar{\beta} / 2}\left(e^{\bar{\omega}\left(\bar{\beta} / 2-\left|\tau-\tau^{\prime}\right|\right)}+e^{-\bar{\omega}\left(\bar{\beta} / 2-\left|\tau-\tau^{\prime}\right|\right)}\right) \sum_{i j} \Phi_{0}\left[\mathbf{r}_{i}(\tau), \mathbf{r}_{j}\left(\tau^{\prime}\right)\right] \\
& +\frac{z \lambda \bar{\omega}}{\Phi_{0}(0,0)} \int_{0}^{\bar{\beta}} \int_{0}^{\bar{\beta}} d \tau d \tau^{\prime} e^{-\bar{\omega} \tau} e^{-\bar{\omega}\left(\bar{\beta}-\tau^{\prime}\right)} \sum_{i j}\left(\Phi_{\Delta}\left[\mathbf{r}_{i}(\tau), \mathbf{r}_{j}\left(\tau^{\prime}\right)\right]-\Phi_{0}\left[\mathbf{r}_{i}(\tau), \mathbf{r}_{j}\left(\tau^{\prime}\right)\right]\right)-\int_{0}^{\beta} V\left(\mathbf{r}_{1}(\tau), \mathbf{r}_{2}(\tau)\right) d \tau
\end{aligned}
$$

The full interaction between the particles is $\Phi_{\Delta \mathbf{r}}\left[\mathbf{r}(\tau), \mathbf{r}\left(\tau^{\prime}\right)\right]=\sum_{\mathbf{m}} f_{\mathbf{m}}[\mathbf{r}(\tau)] f_{\mathbf{m}+\Delta \mathbf{r}}\left[\mathbf{r}\left(\tau^{\prime}\right)\right]$ where the vector $\Delta \mathbf{r}=\mathbf{r}(\beta)-\mathbf{r}(0)$ is the difference between the end points of one of the paths in the non-exchanged configuration (here $\bar{\omega}=\hbar \omega / t$ and $\bar{\beta}=t / k_{B} T$ ). The indices $i=1,2$ and $j=1,2$ represent the fermion paths. $V\left(\mathbf{r}_{1}, \mathbf{r}_{2}\right)$ is an instantaneous Coulomb repulsion. From this starting point, the bipolaron is simulated using the Metropolis Monte-Carlo (MC) method. The electron paths are continuous in time with hopping events (or kinks) introduced or removed from the path with each MC step. Analytic integration is performed over sections of parallel paths. The ends of the two paths at $\tau=0$ and $\tau=\beta$ are related by an arbitrary translation, $\Delta \mathbf{r}$. In contrast to the one-particle case, the fixing of the end configurations limits the update procedure to inserting and removing pairs of kinks and antikinks in the following ways: (a) addition/removal of two kinks to/from different paths, (b) addition/removal of a kink-antikink pair to/from one path, (c) addition and removal of a kink to/from a single path (kink shift), (d) kink addition to one path and antikink removal from 


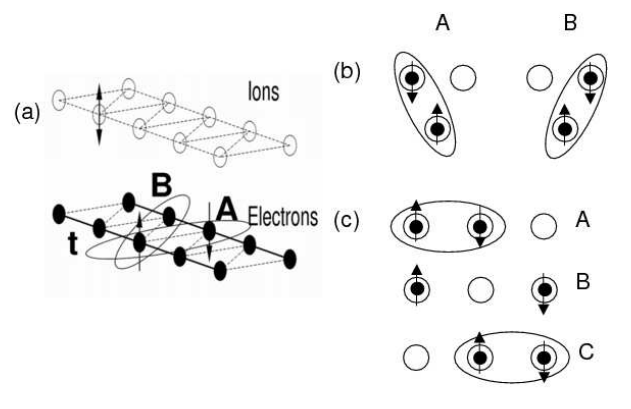

FIG. 1: (a) Schematic of the ladder model. Electrons sit on opposite sides (legs) of a staggered ladder with intersite distance $a$, with ions vibrating across the ladder on an identical system sitting a height $a$ above the electron legs. (b) Schematic motion of the crab bipolaron - the two states are degenerate. (c) Schematic of the crawler motion.

the other path. On kink insertion/removal, either the top or bottom of the path is shifted, which allows the interparticle distance to change. Another significant difference to the one-particle problem is that the paths can now be exchanged. There are two ways to carry out the exchanges: (1) Inserting/removing multiple kinks/antikinks, or (2) If there is a common segment, one may break the paths at that segment, and splice the bottom half of path 1 to the top half of path 2 and viceversa. In the exchanged state, updates (a) to (d) with idential shifts for both single updates are combined with (e) Addition of kink and antikink on different paths (f) Kink addition to 1 path and kink removal from the other (g) Addition of kink and removal of antikink on same path (h) Addition/removal of kink pair to one path. The shift types are opposed for these binary update parts, and allow for a change in the interparticle distance in the exchanged configuration, and as such are essential to sample the full configuration space. From the ensemble the ground state bipolaron energy and effective mass are computed as in Ref. 16]. Also, the bipolaron radius is computed as $R_{b p}=\left\langle\sqrt{\frac{1}{\beta} \int_{0}^{\beta}\left(\mathbf{r}_{1}(\tau)-\mathbf{r}_{2}(\tau)\right)^{2} d \tau}\right\rangle$, where $\beta \gg(\hbar \omega)^{-1}$.

Figure 2 shows the ratio of the polaron to bipolaron masses on the staggered ladder as a function of effective coupling and phonon frequency for $V_{C}=0$. The bipolaron to polaron mass ratio is about 2 in the weak coupling regime $(\lambda \ll 1)$ as it should be for a large bipolaron 3]. In the strong-coupling, large phonon frequency limit the mass ratio approaches 4 , in agreement with strongcoupling arguments given above. In a wide region of parameter space, we find a bipolaron/polaron mass ratio of between 2 and 4 and a bipolaron radius similar to the lattice spacing, see Figs. 3 and 4. Thus the bipolaron is small and light at the same time. Taking into account ad-

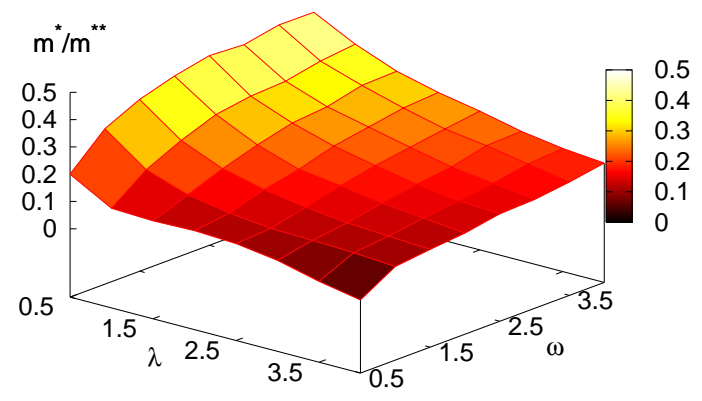

FIG. 2: (color online) Polaron to bipolaron mass ratio for a range of $\bar{\omega}$ and $\lambda$ on the staggered ladder. Mobile small bipolarons are seen even in the adiabatic regime $\bar{\omega}=0.5$ for couplings $\lambda$ up to 2.5 .

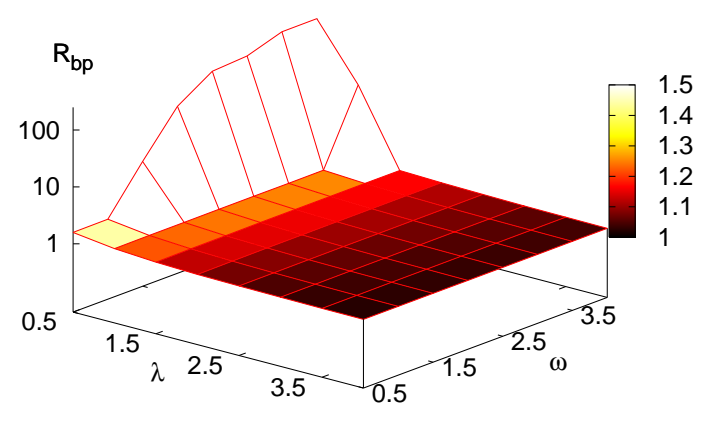

FIG. 3: (color online) Bipolaron radius (in units of $a$ ) for a range of $\bar{\omega}$ and $\lambda$ on the staggered ladder.

ditional intersite Coulomb repulsion $V_{C}$ does not change this conclusion. The bipolaron is stable for $V_{C}<4 t$, see Fig. 4 (inset). As $V_{C}$ increases the bipolaron mass decreases but the radius remains small, at about 2 lattice spacings. Importantly, the absolute value of the small bipolaron mass is only about 4 times of the bare electron mass $m_{0}$, for $\lambda=\hbar \omega / t=1$ (see Fig. 4).

The toy problem on the triangular ladder contains the essential physics of the crab bipolaron. We demonstrate this by simulating the bipolaron on an infinite triangular lattice including exchanges and large on-site Hubbard repulsion $U=20 t$. A moderate coupling $\lambda=0.5$ and a large phonon frequency $\omega=2 t$ lead to $m_{x y}^{* *}=(3.77 \pm 0.04) m_{0 x y}$ and a small bipolaron radius of $(2.056 \pm 0.004) a$. For the triangular lattice, $m_{0 x y}=\hbar^{2} / 3 a^{2} t$. Finally, we have simulated the bipolaron in a hexagonal lattice, with out-of-plane hopping $t^{\prime}=0.3 t$. We have calculated values of the bipolaron mass and radius for experimentally achievable values of the phonon frequency $\omega=t=200 \mathrm{meV}$ and electronphonon coupling $\lambda=0.3$. We have found a light in-plane 


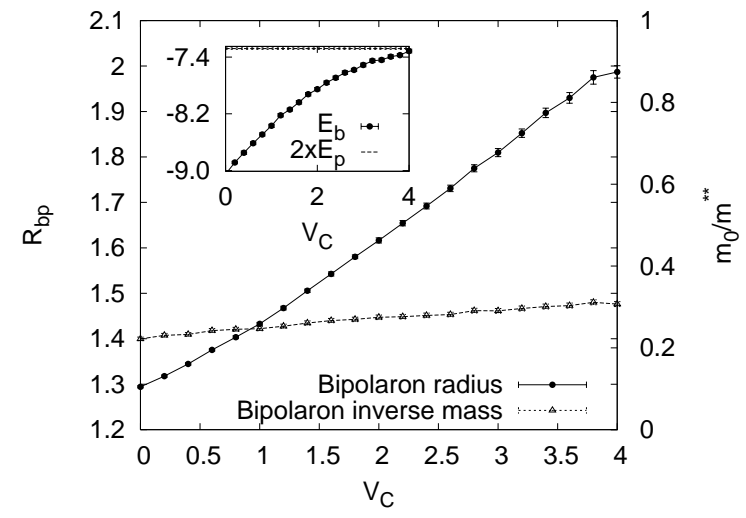

FIG. 4: Variation of bipolaron energy, mass and radius (in units of $a$ ) as intersite Coulomb repulsion $V_{C}$ is increased for $\lambda$ and $\bar{\omega}=1$ on the staggered ladder.

mass, $m_{x y}^{* *}=(4.49 \pm 0.04) m_{0 x y}$. Out-of-plane $m_{z}^{* *}=$ $(68.4 \pm 1) m_{0 z}$ is Holstein like, where $m_{0 z}=\hbar^{2} / 2 d^{2} t^{\prime}$. The bipolaron radius is $R_{b p}=(2.60 \pm 0.03) a$, sitting mainly in the $x y$ plane.

When bipolarons are small and pairs do not overlap, the pairs can form a BEC at $k_{B} T_{\mathrm{BEC}}=$ $3.31 \hbar^{2}\left(2 n_{B} / a^{2} \sqrt{3} d\right)^{2 / 3} /\left(m_{x y}^{* * 2 / 3} m_{z}^{* * 1 / 3}\right)$. If we choose realistic values for the lattice constants of $0.4 \mathrm{~nm}$ in the plane and $0.8 \mathrm{~nm}$ out of the plane, and allow the density of bosons to be $n_{B}=0.12$ per lattice site, which easily avoids overlap of pairs, then $T_{\mathrm{BEC}}=323 \mathrm{~K}$. The longrange Fröhlich interaction combined with Coulomb repulsion might cause clustering of polarons into finite-size quasi-metallic mesoscopic textures. However analytical [15] and QMC [18] studies of mesoscopic textures with lattice deformations and Coulomb repulsion show that pairs (i.e. bipolarons) dominate over phase separation since they effectively repel each other [1].

Recently, there has been a large revival in quantitative studies of polarons owing to evidence for polaronic effects in high-temperature superconductors 19, 20. There are strong arguments in favor of 3D bipolaronic BEC in cuprates [4] drawn using parameter-free fitting of experimental $T_{c}$ with BEC $T_{c}$ 21], unusual upper critical fields and the specific heat [22], and more recently the normal state diamagnetism [23]. Here we have presented the numerically exact bipolaron mass and size, which put these arguments (which require very light bipolarons in the intermediate coupling, moderate phonon-frequency regime) on a solid microscopic ground.

In summary, the CTQMC algorithm to simulate bipolarons in the Coulomb-Fröhlich model has been extended, leading to an unusual bipolaron configuration that is small and superlight. Such a particle has been found in a wide parameter range using CTQMC in triangular lattices with achievable phonon frequencies and couplings in the presence of strong Coulomb repulsion. Such bipolarons could easily have a superconducting transition in excess of room temperature. We believe that the following recipe is worth investigating to look for roomtemperature superconductivity: (a) The parent compound should be an ionic insulator with light ions to form high-frequency optical phonons, (b) The structure should be quasi two-dimensional to ensure poor screening of high-frequency c-axis polarized phonons, (c) A triangular lattice is required in combination with strong, onsite Coulomb repulsion to form the small superlight Crab bipolaron (d) Moderate carrier densities are required to keep the system of small bipolarons close to the dilute regime.

The authors acknowledge support from EPSRC (UK) grants no EP/C518365/1 and no EP/D07777X/1, and useful discussions with J. Devreese, P. Edwards, V. Kabanov, Y. Liang, M. Stoneham, and P. Zhao.

[1] A.S. Alexandrov and N.F. Mott, Rep. Prog. Phys. 57, 1197 (1994).

[2] J.T. Devreese, in Encyclopedia of Physics, Wiley-VHC Publishers (2005).

[3] G. Verbist, F.M. Peeters, and J.T. Devreese, Phys. Rev. B 43, 2712 (1991).

[4] A.S. Alexandrov, Theory of Superconductivity: From Weak to Strong Coupling, IoP Publishing, Bristol (2003).

[5] P.W. Anderson, Phys. Rev. Lett. 34, 953, (1975).

[6] A.S. Alexandrov and J. Ranninger, Phys. Rev. B 23, 1796 (1981).

[7] T. Holstein, Ann. Phys. 8, 325 (1959); ibid, 343 (1959).

[8] E.V.L. de Mello and J. Ranninger, Phys. Rev. B 58, 9098 (1998).

[9] P.W. Anderson, The Theory of Superconductivity in the Cuprates, Princeton University Press, Princeton NY (1997).

[10] A.S. Alexandrov, Phys. Rev. B 53, 2863 (1996).

[11] J.P. Hague et al., Phys. Rev. B 73, 054303 (2006).

[12] A.S. Alexandrov and P.E. Kornilovitch, Phys. Rev. Lett. 82, 807 (1999).

[13] H. Fehske, J. Loos, and G. Wellein, Phys. Rev. B 61, 8016 (2000).

[14] J. Bonča and S.A. Trugman, Phys. Rev. B 64, 094507 (2001).

[15] A.S. Alexandrov and P.E. Kornilovitch, J. Phys.: Cond. Matter 14, 5337 (2002).

[16] P.E. Kornilovitch, Phys. Rev. Lett. 81, 5382 (1998); Phys. Rev. B 60, 3237 (1999).

[17] P.E. Spencer et al., Phys. Rev. B 71, 184310 (2005).

[18] T. Mertelj, V. V. Kabanov, and D. Mihailovic, Phys. Rev. Lett. 94, 147003 (2005).

[19] G. Zhao and D. E. Morris, Phys. Rev. B 51, 16487 (1995);

[20] G-H. Gweon et al., Nature (London) 430, 187 (2004).

[21] A.S. Alexandrov and V.V. Kabanov, Phys. Rev. B 59, 13628 (1999).

[22] V.N. Zavaritsky, V.V. Kabanov and A.S. Alexandrov, Europhys. Lett. 60, 127 (2002).

[23] A.S. Alexandrov, Phys. Rev. Lett. 96, 147003 (2006). 